\title{
Wood Apple Variability - An Underutilized Dry Land Fruit from Gujarat, India
}

\author{
Vikas Yadav ${ }^{1 *}$, Arvind Kumar Singh ${ }^{1}$, Varre Venkata Appa Rao ${ }^{1}$, \\ Sanjay Singh ${ }^{2}$ and Pyare Lal Saroj ${ }^{3}$
}

${ }^{I}$ Department of Fruit Production, Central Horticultural Experiment Station (ICAR-CIAH), Vejalpur-389340, Panchmahals, Gujarat, India

${ }^{2}$ Central Horticultural Experiment Station (ICAR-CIAH), Vejalpur-389340, Panchmahals, Gujarat, India

${ }^{3}$ Central Horticultural Experiment Station (ICAR-CIAH), Beechwal, Bikaner-334006, Rajasthan, India

*Corresponding author

\section{A B S T R A C T}

\section{Keywords}

Wood apple, Genetic diversity, Fruit quality

Article Info

Accepted: 04 May 2018 Available Online: 10 June 2018

\begin{abstract}
Preponderance of wood apple seedlings are seen growing naturally at scattered/isolated areas of various agro climatic zones of Gujarat, India, and chance for the selection of superior genotypes are very high due to huge genetic diversity in the exiting populations. In order to reveal the genetic variability in wood apple, as fruit sample with shoot from diverse areas of province were collected and analysed for various physic-chemical attributes. Results of study revealed wide range of variability found with respect to fruit weight, leaf; spine and fruit quality traits among the studied genotypes. This variability can be exploited for the selection of superior genotype for conservation, evaluation, utilization, and a source for future breeding programme in crop improvement.
\end{abstract}

\section{Introduction}

Dry land farming contributes 67 per cent of the cultivated area, and support to 44 per cent of the population (AICRPDA, 2003). Growing of fruit crops in dryland is known as dryland horticulture. Dryland horticulture in these areas is better option for farmers which provides higher income with lesser use of farm inputs (FAO, 2000). Several fruit crops like ber, pomegranate, custard apple, aonla, guava, tamarind, bael, mahua, chironji and karonda etc. are suitable for cultivation under dryland condition. These crops are inviting keen interest of farmers due to higher stable return with minimum farm input and health security. There are numbers of centres of government and private which are working for development of suitable dryland horticulture farming. Standard agronomic package of practices for many of these dryland fruit crops either sole (bael, jamun, aonla and custard apple) or in agri.-horticultural have been evolved (Hiwale, 2013). By use of in-situ water harvesting techniques, farmers can also get more income from these fruits. The area 
and yield potential of these horticultural crops has increased manifold because of the development of new varieties and agrotechniques in arid region (Sharma and bhargava, 2003).

Wood Apple (Feronia limonia) belongs to the family Rutaceae. It is also known as monkey fruit, curd fruit and kathbel, and native to India and Sri Lanka. The tree prefers dry climatic conditions during flowering and fruit setting. It is one of the very hardy trees and found growing all over drylands areas of India. The plant parts like leaf, stem, bark, fruit, and seed have been used for curing various diseases (Sharma et al., 2012). The fruits contain a myriad of phytochemicals such as polyphenols, phytosterols, saponins, tannins, coumarins, triterpenoids, vitamins, amino acids, tyramine derivatives, etc. (Dar et al., 2013). It also have curative value for various diseases of bones and joints, bilious diseases, prevention of capillary bleeding, piles, dysentery, cold, influenza, habitual constipation and scurvy (Diengngan and Hasan 2015). The ripe fruit pulp makes excellent chutney, and it is also consumed as fresh along with sugar. Its distribution occurs from moist tropical to sub tropical growing countries like Pakistan, Srilanka and South East Asia (Bakshi et al., 2001).

Feronia limonia found all dry part of India, especially in the semi-arid and arid region of Maharashtra, Gujarat, Andhra Pradesh, Karnataka, Tamil Nadu and Madhya Pradesh. With the advent of high yielding varieties of agronomical crops, the diversity found in minor fruit crops is vanishing due to uprooting of indigenous isolated/ scattered growing crops in diversity rich areas particularly in forest. Importance of clonal selections in crop improvement is well recognized by several workers (Badiyala et al., 1992). Therefore, it is necessary to identify superior genotypes of wood apple for their ex-situ collection, conservation, evaluation and utilization in the future breeding programmes. Hence, the present investigations were carried out to record the extent of genetic diversity and locate the elite genotypes possessing desirable horticulture traits.

\section{Materials and Methods}

The present study, morphological characters were studied at their original location, while physic-chemical attributes were carried out at the laboratory of Central Horticultural Experiment Station (ICAR-CIAH), Vejalpur, Panchmahals (Godhra), Gujarat. The diversity rich areas of Gujarat were explored and 39 wood apple genotypes were collected on the basis of preferred horticultural traits like precocity in bearing, less spine intensity, earliness, regular bearing, and dwarf structure of the tree and fruit having strong aroma during 2015-17. Twenty fruits of wood apple were randomly selected from all the direction of marked and selected genotypes, and the bulk of sample of all the selected trees from each site collected then kept into bags and tagged by the number and subjected to physico-chemical analysis in laboratory. Physical parameters such as spine length, and seed size were estimated with the help of digital Vernier Callipers, and fruit weight by electronic weighting machine. Juice percentage was worked out on fresh fruit weight basis. The extracted juice was strained through a muslin cloth, while total soluble solids were noted with Bausch and Lamb hand refractometer in terms of degree brix and values were corrected at $20^{\circ} \mathrm{C}$. Acidity was estimated by titrating $10 \mathrm{ml}$ juice against 0.1 $\mathrm{N} \mathrm{NaOH}$ using phenolphthalein as indicator and ascorbic acid by titration against 2, 6 dichlorophenol indophenol dye (AOAC, 1980). All the selected fruits were analysed for the proximate principle i.e. moisture, protein ( $\mathrm{N} \times$ 6.25), crude fat (ether extraction), ash and crude fibre (NIN, 2003). Fruits sample 
were analysed for calcium, magnesium, iron, zinc and copper through Atomic Absorption Spectrophotometer method suggested by Bishnoi and Brar (1988). The data were statistically analysed as per method outlined by Gomez and Gomez (1984).

\section{Results and Discussion}

The data pertaining to physico-chemical attributes of wood apple exhibited a significant variation with respect to spine, seeds, and fruit quantitative and qualitative characters (Table 1, 2 and 3). The highest variability was recorded for phosphorous $(38.60 \%)$ followed by zinc $(36.17 \%)$, fruit weight $(34.84 \%)$, spine length $(34.20 \%)$ and pectin (25.03). In different genotypes, fruit weight ranged between $145.52 \mathrm{~g}$ to $431.23 \mathrm{~g}$, spine length: $1.20 \mathrm{~cm}$ to $3.47 \mathrm{~cm}$, spine density/meter shoot: 7.62 to 25.23 , number of leaf/meter shoot: 42.38-61.65, seed length: $7.05 \mathrm{~mm}$ to $10.26 \mathrm{~mm}$, seed width: $2.35 \mathrm{~mm}$ to $5.26 \mathrm{~mm}$, seed thickness: $1.31 \mathrm{~mm}$ to 1.70 mm, pectin: $1.03-2.13 \%$, moisture: $70.12 \%$ to $72.92 \%$, fat: $10.23-11.92 \mathrm{mg}$, fibre: $6.20-$ 8.18mg and ash: 4.50-5.31mg.

Variability in chemical attributes was found among different elite selected wood apple genotypes and total soluble solids varied between 13.68 and $21.87{ }^{0}$ Brix, reducing sugars content $1.11-1.30 \%$, and total sugars content $1.92-2.63 \%$. This indicates that elite genotypes may be selected directly from seedling populations on the basis of fruit weight, TSS and mineral content for diverse purpose. Similar variations in fruit characters of wood apple were reported by Singh et al., (2016). The results revealed that, the genotype having more spine length and density may be suitable for dry land areas as root stock; whereas the genotype with less spine length and density/metre shoot may be choose for table and processing purpose like pickle, candy, jelly. In the present study, the relationship of fruit weight and TSS with spine length and spine density indicate that the larger and higher TSS containing fruits have mostly lower spine length and density. The leaf shape of genotypes was cuneate in base and obtuse in apex. The leaf of genotypes has 5 to 9 green color leaflets/leaf. The density of leaf/metre shoot was the highest in CHESW26 (61.65) followed by CHESW- 25 (60.67), CHESW-24 (59.62), CHESW-27 (58.63); however it was minimum in CHESW16(42.38) followed by CHESW- $15 \& 17$ (45.62). Generally, it was observed that the genotype with less spine length and density/metre shoot have higher number of leaf/metre.

The seed size of different genotype varied from 7.05 to $10.26 \mathrm{~mm}$ in length, width 2.35 $5.26 \mathrm{~mm}$ and thickness $1.36-1.70 \mathrm{~mm}$. the maximum seed size $(10.26 \times 5.26 \times 1.70 \mathrm{~mm})$ was found in CHESW- 5 followed by CHESW-9 $(10.22 \times 5.18 \times 1.68 \mathrm{~mm})$ and CHESW-26 (10.16x5.12x1.67mm), and same was found lowest in CHESW-1 $(7.05 \times 2.35 \times 1.34 \mathrm{~mm})$. The maximum TSS was recorded in CHESW-31 $\left(21.87^{\circ}\right.$ Brix $)$ followed by CHESW-37 $\left(21.85^{\circ} \mathrm{Brix}\right)$, CHESW-30 (21.32 ${ }^{\circ}$ Brix $)$ and CHESW-5 $\left(19.27^{0} \mathrm{Brix}\right)$; whereas the lowest was estimated in CHESW-25 (13.68 ${ }^{0}$ Brix) and CHESW 16 (13.72 ${ }^{\circ}$ Brix). The reducing and total sugars were observed the highest in CHESW-24 (1.30\%) and CHESW 16 (2.63\%), and it was found the lowest in CHESW-11 $(1.11 \%)$ and CHESW 21 (1.92\%) respectively. wood apple fruit is rich in pectin hence, it is highly suitable for quality value added products like pickles, jelly etc. pectin content was estimated the highest in CHESW 26 (2.13\%) followed by in CHESW-23(2.11\%), CHESW-28 (2.10\%) and CHESW-35 $(2.05 \%)$, and the same was observed the least in CHESW-3 (1.03\%) followed by CHESW-1 $(1.04 \%)$. On the basis of observations, it was seen that oval fruit had higher pectin than others. 
Table.1 Variation in morphological characters of wood apple from Gujarat, India

\begin{tabular}{|c|c|c|c|c|c|c|c|c|c|}
\hline \multirow[t]{2}{*}{ Genotypes } & \multirow[t]{2}{*}{ Fruit shape } & \multirow{2}{*}{$\begin{array}{c}\text { Spine } \\
\text { Length } \\
(\mathrm{cm})\end{array}$} & \multirow{2}{*}{$\begin{array}{c}\text { Spine } \\
\text { density/ } \\
1 \text { meter } \\
\text { shoot }\end{array}$} & \multirow{2}{*}{$\begin{array}{l}\text { No of } \\
\text { leaf } / 1 \\
\text { meter } \\
\text { shoot }\end{array}$} & \multicolumn{2}{|c|}{ Leaf shape } & \multicolumn{2}{|c|}{$\begin{array}{c}\text { Seed size } \\
(\mathrm{mm})\end{array}$} & \multirow[b]{2}{*}{$\begin{array}{c}\text { Thickn } \\
\text { ess }\end{array}$} \\
\hline & & & & & Base & Apex & Length & Breadth & \\
\hline CHESW-1 & Round & 1.82 & 08.23 & 53.32 & Cuneate & Obtuse & 7.05 & 2.35 & 1.31 \\
\hline CHESW-2 & Round & 2.15 & 11.25 & 54.26 & Cuneate & Obtuse & 7.16 & 2.92 & 1.37 \\
\hline CHESW-3 & Round & 1.92 & 13.15 & 52.56 & Cuneate & Obtuse & 8.37 & 3.12 & 1.64 \\
\hline CHESW-4 & Round & 2.17 & 14.45 & 50.34 & Cuneate & Obtuse & 8.43 & 3.16 & 1.60 \\
\hline CHESW-5 & Round & 1.78 & 12.35 & 55.29 & Cuneate & Obtuse & 10.26 & 5.26 & 1.70 \\
\hline CHESW-6 & Round & 2.32 & 15.21 & 56.45 & Cuneate & Obtuse & 9.23 & 4.36 & 1.62 \\
\hline CHESW-7 & Round & 1.91 & 16.14 & 57.16 & Cuneate & Obtuse & 9.21 & 3.62 & 1.43 \\
\hline CHESW-8 & Round & 2.17 & 13.47 & 54.37 & Cuneate & Obtuse & 9.61 & 3.82 & 1.45 \\
\hline CHESW-9 & Round & 1.76 & 17.26 & 52.75 & Cuneate & Obtuse & 10.22 & 5.18 & 1.68 \\
\hline CHESW-10 & Flattened & 1.45 & 15.15 & 51.45 & Cuneate & Obtuse & 10.14 & 5.10 & 1.65 \\
\hline CHESW-11 & Triangular & 1.87 & 19.21 & 48.35 & Cuneate & Obtuse & 10.08 & 5.05 & 1.60 \\
\hline CHESW-12 & Triangular & 1.95 & 18.12 & 52.29 & Cuneate & Obtuse & 10.12 & 5.00 & 1.61 \\
\hline CHESW-13 & Triangular & 2.10 & 20.16 & 53.62 & Cuneate & Obtuse & 9.21 & 4.78 & 1.50 \\
\hline CHESW-14 & Triangular & 2.65 & 22.18 & 47.46 & Cuneate & Obtuse & 9.26 & 4.35 & 1.42 \\
\hline CHESW-15 & Triangular & 2.45 & 23.24 & 45.62 & Cuneate & Obtuse & 8.36 & 4.62 & 1.51 \\
\hline CHESW-16 & Round & 3.12 & 25.23 & 42.38 & Cuneate & Obtuse & 9.36 & 4.46 & 1.52 \\
\hline CHESW-17 & Oval & 2.45 & 23.28 & 45.46 & Cuneate & Obtuse & 10.12 & 5.20 & 1.62 \\
\hline CHESW-18 & Round & 3.45 & 22.24 & 46.52 & Cuneate & Obtuse & 9.92 & 5.10 & 1.56 \\
\hline CHESW-19 & Round & 3.47 & 20.26 & 48.72 & Cuneate & Obtuse & 9.82 & 4.72 & 1.44 \\
\hline CHESW-20 & Round & 3.35 & 19.31 & 52.48 & Cuneate & Obtuse & 9.73 & 4.78 & 1.46 \\
\hline CHESW-21 & Round & 2.94 & 24.26 & 50.41 & Cuneate & Obtuse & 9.74 & 4.68 & 1.43 \\
\hline CHESW-22 & Oval & 2.70 & 23.35 & 48.34 & Cuneate & Obtuse & 9.85 & 4.56 & 1.62 \\
\hline CHESW-23 & Round & 2.60 & 16.25 & 53.38 & Cuneate & Obtuse & 9.88 & 4.67 & 1.63 \\
\hline CHESW-24 & Round & 1.60 & 08.45 & 59.62 & Cuneate & Obtuse & 7.92 & 4.65 & 1.72 \\
\hline CHESW-25 & Round & 1.40 & 07.62 & 60.67 & Cuneate & Obtuse & 7.96 & 4.52 & 1.54 \\
\hline CHESW-26 & Oval & 1.50 & 09.18 & 61.65 & Cuneate & Obtuse & 10.16 & 5.12 & 1.67 \\
\hline CHESW-27 & Round & 1.80 & 10.37 & 58.63 & Cuneate & Obtuse & 9.27 & 4.25 & 1.48 \\
\hline CHESW-28 & Round & 1.80 & 13.29 & 56.42 & Cuneate & Obtuse & 9.38 & 4.28 & 1.36 \\
\hline CHESW-29 & Round & 1.45 & 15.85 & 57.49 & Cuneate & Obtuse & 9.24 & 4.00 & 1.52 \\
\hline CHESW-30 & Round & 2.70 & 12.16 & 49.43 & Cuneate & Obtuse & 7.57 & 2.36 & 1.47 \\
\hline CHESW-31 & Round & 1.20 & 16.24 & 46.48 & Cuneate & Obtuse & 8.19 & 4.2 & 1.43 \\
\hline CHESW-32 & Flattened & 2.13 & 13.37 & 47.32 & Cuneate & Obtuse & 8.17 & 4.08 & 1.40 \\
\hline CHESW-33 & Flattened & 3.01 & 21.31 & 55.37 & Cuneate & Obtuse & 7.76 & 4.13 & 1.57 \\
\hline CHESW-34 & Round & 1.70 & 15.19 & 53.36 & Cuneate & Obtuse & 7.83 & 3.83 & 1.52 \\
\hline CHESW-35 & Round & 1.60 & 17.17 & 54.24 & Cuneate & Obtuse & 7.62 & 4.31 & 1.42 \\
\hline CHESW-36 & Round & 1.80 & 13.43 & 58.16 & Cuneate & Obtuse & 8.56 & 4.11 & 1.50 \\
\hline CHESW-37 & Round & 2.10 & 16.56 & 54.18 & Cuneate & Obtuse & 8.13 & 3.12 & 1.44 \\
\hline CHESW-38 & Round & 2.30 & 17.45 & 52.45 & Cuneate & Obtuse & 7.42 & 3.26 & 1.42 \\
\hline CHESW-39 & Round & 1.80 & 18.75 & 50.42 & Cuneate & Obtuse & 7.36 & 2.86 & 1.49 \\
\hline C.D. $(0.05)$ & 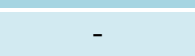 & 0.401 & 1.610 & 5.210 & - & - & 0.89 & 0.42 & 0.15 \\
\hline
\end{tabular}




\section{Table.2 Variations in quality characters of wood apple from Gujarat, India}

\begin{tabular}{|c|c|c|c|c|c|c|c|c|c|c|}
\hline Genotypes & $\begin{array}{c}\text { Fruit } \\
\text { weight (g) }\end{array}$ & $\begin{array}{c}\text { TSS } \\
\left({ }^{\circ} \text { Brix }\right)\end{array}$ & $\begin{array}{c}\text { Reducin } \\
\text { g sugars } \\
(\%)\end{array}$ & $\begin{array}{c}\text { Total } \\
\text { sugars } \\
(\%)\end{array}$ & $\begin{array}{c}\text { Pectin } \\
(\%)\end{array}$ & $\begin{array}{l}\text { Moistu } \\
\text { re (\%) }\end{array}$ & $\begin{array}{c}\text { Fat }(\mathrm{mg} / \\
100)\end{array}$ & $\begin{array}{l}\text { Fibre } \\
\text { (mg) }\end{array}$ & $\begin{array}{c}\text { Ash } \\
\text { (mg) }\end{array}$ & Juice $\%$ \\
\hline CHESW-1 & 145.52 & 14.23 & 1.12 & 1.98 & 1.04 & 70.34 & 11.37 & 7.48 & 5.20 & 15.18 \\
\hline CHESW-2 & 183.35 & 16.28 & 1.25 & 2.40 & 1.08 & 70.4 & 11.21 & 6.35 & 4.80 & 15.12 \\
\hline CHESW-3 & 188.24 & 17.16 & 1.18 & 2.36 & 1.03 & 72.25 & 11.26 & 6.45 & 4.82 & 15.23 \\
\hline CHESW-4 & 195.52 & 18.22 & 1.17 & 2.27 & 1.09 & 70.46 & 11.92 & 8.10 & 5.28 & 18.20 \\
\hline CHESW-5 & 301.45 & 19.27 & 1.22 & 2.27 & 1.82 & 70.82 & 10.96 & 8.08 & 5.21 & 17.26 \\
\hline CHESW-6 & 201.36 & 15.25 & 1.12 & 2.14 & 1.72 & 72.38 & 10.86 & 6.96 & 4.92 & 16.12 \\
\hline CHESW-7 & 214.45 & 17.22 & 1.20 & 2.07 & 1.60 & 71.25 & 11.35 & 6.90 & 4.93 & 15.00 \\
\hline CHESW-8 & 134.78 & 18.29 & 1.19 & 2.43 & 1.52 & 71.65 & 11.32 & 6.20 & 4.50 & 17.26 \\
\hline CHESW-9 & 307.71 & 16.26 & 1.24 & 2.53 & 1.45 & 72.62 & 11.86 & 8.12 & 5.20 & 18.26 \\
\hline CHESW-10 & 431.23 & 17.24 & 1.20 & 2.35 & 1.36 & 72.92 & 11.80 & 8.05 & 5.38 & 18.80 \\
\hline CHESW-11 & 189.53 & 16.22 & 1.23 & 2.42 & 1.20 & 70.23 & 10.82 & 6.37 & 4.96 & 15.52 \\
\hline CHESW-12 & 254.24 & 14.21 & 1.11 & 2.19 & 1.12 & 70.12 & 10.88 & 6.82 & 4.98 & 16.20 \\
\hline CHESW-13 & 268.82 & 19.18 & 1.17 & 2.31 & 1.07 & 70.28 & 11.22 & 6.73 & 4.89 & 16.23 \\
\hline CHESW-14 & 318.42 & 16.31 & 1.19 & 2.37 & 1.33 & 71.92 & 11.75 & 7.95 & 5.39 & 17.26 \\
\hline CHESW-15 & 284.62 & 17.52 & 1.21 & 2.41 & 1.36 & 70.34 & 10.23 & 6.72 & 4.78 & 17.36 \\
\hline CHESW-16 & 320.45 & 13.73 & 1.22 & 2.63 & 1.92 & 71.56 & 11.70 & 7.80 & 5.29 & 17.20 \\
\hline CHESW-17 & 330.32 & 14.22 & 1.16 & 2.61 & 1.47 & 71.62 & 11.78 & 7.83 & 5.31 & 16.86 \\
\hline CHESW-18 & 300.12 & 16.56 & 1.14 & 2.43 & 1.53 & 71.69 & 11.80 & 7.96 & 5.26 & 16.82 \\
\hline CHESW-19 & 245.32 & 15.21 & 1.19 & 2.11 & 1.56 & 70.82 & 11.32 & 6.68 & 4.82 & 16.80 \\
\hline CHESW-20 & 283.45 & 16.38 & 1.21 & 2.51 & 1.63 & 70.36 & 10.70 & 6.52 & 4.90 & 16.81 \\
\hline CHESW-21 & 224.35 & 17.32 & 1.12 & 1.92 & 1.67 & 70.26 & 10.78 & 6.42 & 5.12 & 16.70 \\
\hline CHESW-22 & 275.25 & 16.23 & 1.17 & 2.05 & 1.70 & 70.15 & 10.56 & 6.78 & 5.10 & 16.20 \\
\hline CHESW-23 & 292.30 & 17.26 & 1.23 & 2.08 & 2.11 & 70.18 & 10.72 & 6.83 & 5.05 & 16.86 \\
\hline CHESW-24 & 248.21 & 15.23 & 1.30 & 2.33 & 1.92 & 71.2 & 10.93 & 6.85 & 5.07 & 16.92 \\
\hline CHESW-25 & 280.26 & 13.68 & 1.18 & 2.40 & 1.82 & 71.22 & 10.66 & 6.92 & 5.17 & 17.35 \\
\hline CHESW-26 & 153.56 & 17.52 & 1.15 & 2.42 & 2.13 & 70.17 & 10.73 & 6.23 & 4.70 & 15.10 \\
\hline CHESW-27 & 170.25 & 19.34 & 1.20 & 2.47 & 2.05 & 70.90 & 10.23 & 6.27 & 4.60 & 16.23 \\
\hline CHESW-28 & 375.36 & 17.26 & 1.23 & 2.48 & 2.10 & 71.86 & 11.82 & 8.18 & 5.35 & 18.18 \\
\hline CHESW-29 & 270.62 & 14.92 & 1.12 & 2.09 & 1.92 & 71.23 & 10.56 & 8.00 & 5.15 & 16.29 \\
\hline CHESW-30 & 288.72 & 21.32 & 1.19 & 2.17 & 1.96 & 70.96 & 10.62 & 6.38 & 5.12 & 16.82 \\
\hline CHESW-31 & 285.82 & 21.87 & 1.14 & 2.27 & 1.37 & 70.68 & 10.78 & 6.48 & 5.14 & 16.70 \\
\hline CHESW-32 & 183.93 & 18.60 & 1.16 & 2.32 & 1.39 & 70.46 & 10.50 & 6.38 & 4.99 & 16.38 \\
\hline CHESW-33 & 185.63 & 14.13 & 1.20 & 2.34 & 1.29 & 70.68 & 10.45 & 6.42 & 5.23 & 16.88 \\
\hline CHESW-34 & 228.26 & 17.32 & 1.17 & 2.19 & 1.19 & 70.46 & 10.52 & 6.72 & 4.72 & 16.53 \\
\hline CHESW-35 & 256.32 & 14.65 & 1.15 & 2.24 & 2.05 & 70.31 & 10.55 & 6.80 & 4.78 & 16.57 \\
\hline CHESW-36 & 259.32 & 19.23 & 1.20 & 2.12 & 1.87 & 70.27 & 10.66 & 6.79 & 4.90 & 16.87 \\
\hline CHESW-37 & 268.24 & 21.85 & 1.19 & 2.19 & 1.76 & 70.52 & 11.09 & 6.74 & 4.76 & 16.73 \\
\hline CHESW-38 & 280.56 & 16.58 & 1.24 & 2.39 & 1.72 & 71.16 & 10.84 & 6.92 & 4.83 & 16.83 \\
\hline CHESW-39 & 220.72 & 17.32 & 1.17 & 2.12 & 1.32 & 70.20 & 10.58 & 6.23 & 5.13 & 16.12 \\
\hline C.D. $(0.05)$ & 49.62 & 01.88 & 0.12 & 0.15 & 0.15 & 7.09 & 1.10 & 0.70 & 0.50 & 1.67 \\
\hline
\end{tabular}




\begin{tabular}{|c|c|c|c|c|c|c|c|c|}
\hline Genotypes & $\begin{array}{c}\text { Nitrogen } \\
(\%)\end{array}$ & $\begin{array}{c}\text { Phosphorus } \\
(\%)\end{array}$ & $\begin{array}{c}\text { Potassium } \\
(\%)\end{array}$ & $\begin{array}{c}\text { Calcium } \\
(\%)\end{array}$ & $\begin{array}{c}\text { Magnesium } \\
(\%)\end{array}$ & $\begin{array}{l}\text { Iron } \\
\text { (mg) }\end{array}$ & $\begin{array}{l}\text { Zinc } \\
\text { (mg) }\end{array}$ & $\begin{array}{c}\text { Copper } \\
\text { (mg) }\end{array}$ \\
\hline CHESW-1 & 3.32 & 0.029 & 1.20 & 0.12 & 0.3 & 14.64 & 2.65 & 0.35 \\
\hline CHESW-2 & 3.28 & 0.030 & 1.21 & 0.11 & 0.28 & 14.60 & 2.30 & 0.30 \\
\hline CHESW-3 & 3.42 & 0.038 & 1.22 & 0.19 & 0.31 & 14.72 & 2.43 & 0.33 \\
\hline CHESW-4 & 3.56 & 0.042 & 1.27 & 0.15 & 0.46 & 14.73 & 2.46 & 0.31 \\
\hline CHESW-5 & 4.75 & 0.068 & 1.82 & 0.27 & 0.56 & 14.82 & 2.52 & 0.36 \\
\hline CHESW-6 & 3.62 & 0.047 & 1.41 & 0.18 & 0.50 & 15.12 & 2.62 & 0.32 \\
\hline CHESW-7 & 3.41 & 0.052 & 1.52 & 0.20 & 0.45 & 15.83 & 2.65 & 0.33 \\
\hline CHESW-8 & 3.92 & 0.049 & 1.48 & 0.30 & 0.60 & 15.72 & 2.16 & 0.37 \\
\hline CHESW-9 & 4.68 & 0.062 & 1.74 & 0.32 & 0.52 & 15.60 & 2.28 & 0.31 \\
\hline CHESW-10 & 4.92 & 0.070 & 1.73 & 0.23 & 0.53 & 15.32 & 2.12 & 0.32 \\
\hline CHESW-11 & 4.22 & 0.037 & 1.53 & 0.30 & 0.43 & 15.28 & 2.17 & 0.31 \\
\hline CHESW-12 & 4.32 & 0.034 & 1.47 & 0.25 & 0.42 & 15.18 & 2.34 & 0.32 \\
\hline CHESW-13 & 4.41 & 0.038 & 1.56 & 0.21 & 0.52 & 14.82 & 2.50 & 0.33 \\
\hline CHESW-14 & 4.72 & 0.063 & 1.84 & 0.26 & 0.50 & 14.32 & 2.47 & 0.30 \\
\hline CHESW-15 & 4.22 & 0.056 & 1.43 & 0.18 & 0.43 & 14.52 & 2.37 & 0.33 \\
\hline CHESW-16 & 4.12 & 0.046 & 1.39 & 0.16 & 0.63 & 14.63 & 2.21 & 0.32 \\
\hline CHESW-17 & 4.80 & 0.066 & 1.67 & 0.24 & 0.49 & 14.68 & 2.31 & 0.33 \\
\hline CHESW-18 & 4.56 & 0.064 & 1.63 & 0.15 & 0.50 & 16.12 & 2.26 & 0.20 \\
\hline CHESW-19 & 4.62 & 0.060 & 1.72 & 0.17 & 0.29 & 16.23 & 2.44 & 0.29 \\
\hline CHESW-20 & 4.78 & 0.067 & 1.66 & 0.29 & 0.47 & 16.10 & 2.18 & 0.27 \\
\hline CHESW-21 & 4.80 & 0.057 & 1.53 & 0.19 & 0.43 & 16.22 & 2.29 & 0.32 \\
\hline CHESW-22 & 4.83 & 0.052 & 1.64 & 0.15 & 0.49 & 16.10 & 2.48 & 0.33 \\
\hline CHESW-23 & 4.93 & 0.067 & 1.82 & 0.31 & 0.55 & 16.54 & 3.50 & 0.36 \\
\hline CHESW-24 & 3.96 & 0.058 & 1.58 & 0.14 & 0.56 & 14.15 & 5.54 & 0.27 \\
\hline CHESW-25 & 3.85 & 0.042 & 1.64 & 0.12 & 0.29 & 15.20 & 2.60 & 0.28 \\
\hline CHESW-26 & 4.72 & 0.041 & 1.72 & 0.17 & 0.39 & 15.20 & 2.63 & 0.26 \\
\hline CHESW-27 & 4.46 & 0.033 & 1.43 & 0.29 & 0.37 & 15.36 & 2.13 & 0.25 \\
\hline CHESW-28 & 5.10 & 0.068 & 1.59 & 0.31 & 0.46 & 15.28 & 2.42 & 0.24 \\
\hline CHESW-29 & 4.87 & 0.053 & 1.52 & 0.26 & 0.49 & 16.32 & 2.32 & 0.26 \\
\hline CHESW-30 & 4.93 & 0.054 & 1.74 & 0.24 & 0.50 & 16.45 & 2.70 & 0.27 \\
\hline CHESW-31 & 5.07 & 0.044 & 1.82 & 0.20 & 0.38 & 14.92 & 2.18 & 0.28 \\
\hline CHESW-32 & 4.64 & 0.032 & 1.83 & 0.19 & 0.36 & 14.96 & 2.13 & 0.29 \\
\hline CHESW-33 & 4.36 & 0.039 & 1.57 & 0.25 & 0.33 & 15.36 & 2.53 & 0.23 \\
\hline CHESW-34 & 4.49 & 0.059 & 1.35 & 0.19 & 0.38 & 15.46 & 2.57 & 0.31 \\
\hline CHESW-35 & 4.64 & 0.051 & 1.38 & 0.23 & 0.46 & 15.56 & 2.47 & 0.26 \\
\hline CHESW-36 & 4.57 & 0.041 & 1.34 & 0.21 & 0.42 & 15.28 & 2.35 & 0.222 \\
\hline CHESW-37 & 4.87 & 0.031 & 1.67 & 0.15 & 0.32 & 16.92 & 2.33 & 0.24 \\
\hline CHESW-38 & 5.12 & 0.069 & 1.64 & 0.28 & 0.51 & 16.23 & 2.22 & 0.26 \\
\hline CHESW-39 & 5.04 & 0.040 & 1.67 & 0.18 & 0.29 & 16.63 & 2.08 & 0.23 \\
\hline C.D. $(0.05)$ & 0.34 & 0.010 & 0.13 & 0.047 & 0.08 & 1.540 & 0.25 & 0.03 \\
\hline
\end{tabular}


The juice content of different genotypes showed significant variability and it was found the maximum in CHESW-10 (18.80\%) followed by CHESW-9 (18.26\%), CHESW-4 (18.20\%), CHESW-28 (18.18\%); and same was the least in CHESW-7 (15.00\%) followed by CHESW26 (15.10), CHESW-2 (15.12\%). The maximum fat content in fruit was recorded in CHESW-4 (11.92mg) followed by CHESW-9 (11.86mg), CHESW-10\&18 (11.80mg), CHESW-17 (11.78 $\mathrm{mg}$ ) and it was the lowest in CHESW-15\&27 (10.23mg) followed by CHESW-33 (10.45mg), CHESW-34 (10.52mg) and CHESW-35 $(10.55 \mathrm{mg})$. wood apple also have good amount of fibre content, which was varied between $6.20 \mathrm{mg}-8.18 \mathrm{mg}$ being the highest in CHESW$28(8.18 \mathrm{mg})$ followed by CHESW-9 $(8.12 \mathrm{mg})$, CHESW-4 (8.10mg), CHESW-5 (8.08mg) and CHESW-10 (8.05mg); however, it was observed the lowest in CHESW-8(6.20mg) followed by CHESW-26\&29 (6.23mg), CHESW-27 (6.27mg) and CHESW-2 (6.35mg). The highest ash content was recorded in CHESW-14 (5.39mg) followed by CHESW-10 (5.38mg), CHESW-20 (5.35mg), CHESW-17 $(5.31 \mathrm{mg})$, and it was found lowest in CHESW-8 (4.50mg) followed by CHESW-27 (4.60mg) and CHESW-26 (4.70mg).

Mineral content in different genotypes varied significantly like N 3.28-5.12\%, P $0.029-0.69 \%$, $\mathrm{K} 1.20-1.82$, Ca $0.11-0.32 \%, \mathrm{Mg} 0.28-0.63 \%$, $\mathrm{Fe}$ 14.15-16.92 mg, Zn 2.12-3.54 mg, and $\mathrm{Cu}$ $0.20-0.37 \mathrm{mg}$. The maximum $\mathrm{N}$ content $(5.12 \%)$ was recorded the CHESW-38 followed by CHESW-28 (5.10\%), CHESW-31 (5.07\%) and CHESW-39 (5.04mg); however it was the lowest in CHESW-2 (3.32\%) followed by CHESW-1 (3.32\%) and CHESW-3 (3.42\%). CHESW-30 (0.069\%) exhibited the highest phosphorous content followed by CHESW-20 $(0.068 \%)$, while lowest was observed in CHESW-1 (0.029\%). The potassium content was found highest in CHESW-14 (1.84\%) followed by CHESW-32 (1.83\%), CHESW$31 \& 23(1.82 \%)$ and same was found least in CHESW-1 (1.20\%) and CHESW-2 (1.21\%). The calcium was estimated the highest in CHESW-9 $(0.32 \%)$ followed by CHESW-
$23 \& 28(0.31 \%)$ and CHESW-11\&8 (0.30\%); while it was the least in CHESW-2 $(0.11 \%)$ followed by CHESW- $2725(0.12 \%)$ and CHESW-24 (0.14\%). The maximum $\mathrm{Mg}$ content was recorded in CHESW-16 (0.63\%) followed by CHESW-8 (0.60\%), CHESW-5 $(0.56 \%)$ and CHESW-23 $(0.55 \%)$, and same was the lowest in CHESW-2 (0.28\%) followed by CHESW-25\&39 (0.29\%), CHESW-1 $(0.30 \%)$ and CHESW-3 (0.31\%).

Iron content in wood apple found maximum in CHESW-37 (16.92mg) followed by CHESW39 (16.63 mg), CHESW-23 (16.54mg) and CHESW-30 (16.45); however it was least in CHESW-24 (14.15mg) followed by CHESW14 (14.32mg), CHESW-15 (14.52mg) and CHESW-16 (14.63mg).

The highest $\mathrm{Zn}$ content was recorded in CHESWa-24 (3.54mg) followed by CHESW-23 (3.50mg), CHESW-30 (2.70mg) and CHESW-7 $(2.65 \mathrm{mg})$ and found lowest in CHESW-10 (2.12mg) followed by CHESW-27 (2.13mg), CHESW-8) 2.16mg) and CHESW-11 (2.17mg). $\mathrm{Cu}$ was recorded high in CHESW-8 $(0.37 \mathrm{mg})$ followed by CHESW-(0.36mg), and was the least in CHESW-18 (0.20mg) followed by CHESW-33 (0.23mg) and CHESW-37 $(0.24 \mathrm{mg})$. The genotype (CHESW-23) was found better in mineral content than other genotypes. The finding in line with the results has been reported by Ghosh et al., (2016) in wood apple and Singh and Singh (2005) in jamun.

Based on various observations, it may inferred from the studied that wood apple genotypes exhibited enormous genetic diversity in the existing population of rainfed areas of Gujarat with respect to morphological and quality traits. This species is rich in nutritional and medicinal value and tribals of state prefer this fruit in their diet. Therefore, collection, conservation and evaluation of wood apple can be useful for selection of elite genotype and also may be source for crop improvement in future breeding program. 


\section{References}

AICRPDA 2003 All India Coordinated Research Project for Dryland Agriculture Central Research Institute for Dryland Agriculture report. Pp. 200-210.

Badiyala, S. D., Lakhanpal, S, C, and Bhargava, J, N. 1992. Seedless selection of kagzi lime. Indian Horticulture. 37(1): 95-97

Bakshi, D. N. G., Sensarma, P., Pai, D. C. 2001. A Lexicon of Medicinal Plants in India, Naya Prokash, Calcutta, 2001, 186-187

Bishnoi, R. K. and Brar, S. P. C. 1988. A handbook of soil testing. Printed and published by Punjab Agricultural University. Ludhiyana.

Dar, A. I., Masar, G., Jadhaw, V., Bansal, S. K. and Saxena, R. C. 2013. Isolation and structural elucidation of the novel flavone glycoside from Feronia limonia L. Journal of Pharmacy Research 7: 697704.

Diengngan, S. and Hasan, M. A. 2015. Genetic Diversity of Underutilized Fruits in India for Environmental Sustainability. Adv Plants Agric Res 2015, 2(7): 00076

FAO. 2000. Crops and Drops. Rome, Italy

Ghosh, S. N., Banik, A. K. and Banik, B. C. 2011. Conservation, Multiplication and Utilization of wood apple (Feronia limonia) -a semi-wild fruit crop in West Bengal (India), International Symposium on Minor Fruits and Medicinal Plants, the farmer's training centre in kalyani, Nadia. Bidhan Chandra Krishi
Viswavidyalaya, West Bengal, 19-22 December 2011: 1208-1214.

Gomez, K. A., and Gomez, A. A. 1984. Statistical Procedure for Agricultural Research, 2nd edn. John Wiley and Sos Inc., New York.

Hiwale, S. S. 2013. Annual report, CIAH, Bikaner, India.

NIN 2003. A manual of laboratory techniques. Edited by Raghuramula, N., Nair, K. and Kalyanasunderam, S. (Eds.). National Institute of Nutrition. ICMR, Hyderabad.

Pardeep Singh Shehrawat and Sube Singh 2003. Management of dryland sustainable agriculture. International farm management congress 2003.

Sharma, P., Bodhankar, S, L, and Thakurdesai, P, A. 2012. Protective effect of aqueous extract of Feronia elephantum Correa leaves on thioacetamide induced liver necrosis in diabetic rats. Asian Pacific Journal of Tropical Biomedicine 2(9): 691-5.

Sharma, S. K., Singh, R. S. and Bhargava, R. 2013. Arid Horticulture: An Overview, Annals of Arid Zone 52(3\&4): 251-264.

Singh, A. K., Singh, S., Yadav, V. and Sharma, B. D. 2016. Genetic variability in wood apple (Feronia limonia) from Gujarat. Indian J. Agric. Sci. 86 (11): 1504-1508.

Singh, S. and Singh, A. K. 2005.Genetic variability in jamun (Syzygium cumini) in Gujarat. Progressive Horticulture 37 (1): 144-8.

\section{How to cite this article:}

Vikas Yadav, Arvind Kumar Singh, Varre Venkata Appa Rao, Sanjay Singh and Pyare Lal Saroj. 2018. Wood Apple Variability - An Underutilized Dry Land Fruit from Gujarat, India. Int.J.Curr.Microbiol.App.Sci. 7(06): 548-555. doi: https://doi.org/10.20546/ijcmas.2018.706.063 